\title{
Wie alles anfing, oder „Hinter den Kulissen von netbib“*
}

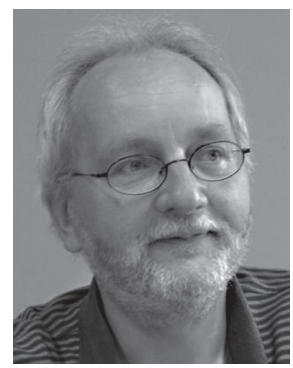

Der Autor, Bibliothekar im Servicebereich der Universitätsbibliothek der TU Hamburg-Harburg, lässt die Entwicklung der bibliothekarischen Website netbib Revue passieren. Hauptaugenmerk wird auf das netbib Weblog gerichtet, das erste seiner Art in Deutschland. Aber auch die weiteren Angebote wie die netbib Wikis und der netbib Newsletter werden vorgestellt. Das netbib Weblog existiert jetzt seit über sechs Jahren und ist für viele an Bibliotheken Interessierte zu einem wichtigen Informationsmittel geworden.

Zurzeit schreiben 15 Autorlnnen in dem Weblog und berichten täglich über aktuelle Entwicklungen aus der bibliothekarischen Praxis im In- und Ausland.

How it all began, or „behind the scenes of netbib“

The author, librarian of the University Library of TU Hamburg-Harburg, lines out the history, development and technical backgrounds of the library focussed website netbib. The netbib weblog, as the essential part of netbib, has been the first of its kind in Germany. netbib is also offering further services like the netbib wikis and the netbib newsletter. The netbib weblog has been established six years ago, and for many librarians and libraries enthusiasts is has become an important source of information.

At the moment 15 authors are contributing to the weblog. They cover current developments in library science as well as best practice examples.

Comment tout a commencé, ou „dans les coulisses de netbib“

L'auteur, conseiller à la documentation à la bibliothèque universitaire de l'Université Technique de Hambourg-Harbourg, relate le développement de netbib, site web pour bibliothèques. L'accent est mis sur le weblog netbib, pionnier dans son genre en Allemagne. L'article présente également les autres services tels que les wikis et la newsletter spécifiques à netbib. Existant depuis plus de six ans, le weblog netbib est devenu aujourd'hui un important outil d'information pour la plupart de ceux qui s'intéressent aux bibliothèques.

L'équipe du weblog compte actuellement 15 auteurs qui informent quotidiennement des nouveautés survenant dans les bibliothèques en Allemagne et à l'étranger. 
Falls Sie nicht wissen, was ein Weblog eigentlich ist, hier eine Definition aus Meyers Lexikon online.

„Weblog [zu englisch web >Netzı und Log(buch)] das, Kurzform Blog, im Internet geführtes Tagebuch, in dem z. B. aktuelle, dem WWW entnommene Informationen chronologisch aufgelistet und regelmäßig aktualisiert werden, versehen mit weiterführenden Informationen und kommentierten Links (Hypertexte) zu anderen Webseiten. Die meisten Weblogs haben eine Kommentarfunktion, die es den Nutzern ermöglicht, ihrerseits einen Eintrag zu kommentieren und mit dem Autor (১Bloggerı) oder anderen Lesern in Dialog zu treten."

Netbib spielt bei der Dokumentation von neuen Entwicklungen im Bibliotheksbereich eine relativ wichtige Rolle. Neue Entwicklungen und Projekte wurden nicht nur vorgestellt und teilweise kritisch begleitet, sondern sicher auch angestoßen.

Netbib ist nicht nur, aber doch zu einem großen Teil das netbib Weblog ${ }^{2}$. Wenn Sie wissen möchten, wie es entstanden ist, dann sind Sie hier richtig. Mindestens genauso interessant sind die Nebeneffekte, die das Webloglesen und -schreiben bei mir und anderen hervorgerufen hat. Das Medium Weblog spielte im bibliothekarischen Bereich in Deutschland lange nur eine untergeordnete Rolle. Es wurde nicht ernst genommen. Während in anderen Ländern das Thema (bibliothekarische) Weblogs seit längerem nicht nur in der Fachliteratur und mit eigenen Konferenzen breiten Raum einnimmt, wird es bei uns erst durch den Begriff Library 2.0 bzw. Bibliothek 2.0 und durch das Schlagwort „social software“ wahrgenommen.

So war auf dem Bibliothekskongress in Leipzig im März 2007 die Veranstaltung „Bibliotheken und soziale Software" total überlaufen, und auch Fortbildungsangebote zum Thema Weblogs, Wikis und was noch so dazugehört sind aktuell meist nach kürzester Zeit ausgebucht. Dennoch, die „breite Masse“ der KollegInnen hat das Thema noch nicht erreicht, vielleicht ändert ja dieser Beitrag etwas daran.

Viele bibliothekarisch relevante Themen haben in netbib ihren Niederschlag gefunden und damit manchmal auch ihren Anfang für den Einsatz im deutschen Bibliothekswesen.

Beispiele gefällig?

- RSS-Feeds für die aktive Nutzerinformation/Marketing

Nur eine lächerlich geringe Anzahl deutscher Bibliotheken (geschätzt etwa 30-40) bietet ihre Neuigkeiten als RSS-Feed an. In anderen Ländern ist dies dagegen Standard auf Bibliothekswebseiten. Auch das z. B. Neuerwerbungslisten fachspezifisch angeboten werden, ist bei uns keineswegs selbstverständlich. Es gibt hier seit einiger Zeit Bibliotheken, die an solchen Diensten arbeiten. Eine Übersicht zu dem Thema findet sich im netbib-Wiki ${ }^{3}$.

- Einsatz von Wikis im bibliothekarischen Bereich Lange vor dem GBV-Wiki gab es die netbib-Wikis, in denen gemeinsam oder allein Informationen und Ideen abgelegt und entwickelt wurden.

- Vorstellung der Firefox-Suchplugins

Suchplugins sind praktisch und sehr einfach für den eigenen Bibliothekskatalog zu kreieren. Sie sind aber auch eine sehr gute Möglichkeit, sich beim Vermarkten der Bibliotheksdienstleistungen als innovative Institution darzustellen. Eine Sammelstelle für diese Plugins ist das buecherei-Wiki ${ }^{4}$.
- Einführung von CMS in Bibliotheken mit Erfahrungsberichten

- Einführung von Chatauskunft in Bibliotheken

Das erstmals im netbib Weblog besprochene (und von der Universitätsbibliothek der Technischen Universität Hamburg Harburg modifizierte) Chat-Auskunftsprogramm RAKIM wird inzwischen von den meisten deutschen Bibliotheken, die eine solche Auskunft anbieten, eingesetzt.

- Fortlaufende Informationen über Bibliothekskataloge und deren Anreicherung

- Tagging in Bibliothekskatalogen und Sacherschließung in und durch soziale Netzwerke

Der Spezialist für dieses Thema, Lambert Heller, schreibt im netbib Weblog sehr umfassend zu diesem sehr wichtigen - im deutschsprachigen Raum vernachlässigten - Bereich der Einbeziehung von Benutzern bei diesem traditionell den Fachreferenten vorbehaltenen Arbeitsgang. Es ist wohl nicht mehr zeitgemäß, darauf zu hoffen, dass die normalen Bibliotheksbenutzer tatsächlich nur darauf warten, von Spezialisten (Fachreferenten) zusammengestellte Informationen zu konsumieren. Wie Heller es nennt: „Der explorativ vorgehende, selbstlernende, vernetzt arbeitende Informationsbenutzer betritt die Szene"5.

Knapp 18000 Einträge gibt es in netbib. Sie sind erschlossen durch eine Suchfunktion, Kategorien und seit ca. 1,5 Jahren auch durch Tagging.

Doch entspricht dies dem Wissensstand in den Bibliotheken?

In Deutschland findet die Fort- und Weiterbildung im eigenen Fach immer noch so statt, wie man es mal im Studium gelernt hat:

- Sichtung der neueren Monographien

- Sichtung der aktuellsten Zeitschriftenliteratur

- Besuch von Konferenzen und Tagungen.

Doch gerade neue aufregende Entwicklungen tauchten in den letzten Jahren nur sehr spärlich in den (deutschen) bibliothekarischen Fachzeitschriften auf, während in netbib - und inzwischen auch in weiteren Weblogs - schon seit Jahren darüber berichtet wird.

Warum findet man nun primär in Weblogs die interessanten Informationen?

Ein Weblogeintrag eignet sich ausgezeichnet für Informationen, die für einen Artikel (noch) nicht reichen, zum Beispiel, weil sie so neu sind. Es hat sich gezeigt, dass

Dieser Artikel ist lizensiert unter der Creative Commons Attribution 2.0 Germany License <http://creativecommons. org/licenses/by/2.0/de/>.

Meyers Lexikonverlag, Definition Weblog: <http://lexikon. meyers. de/index. php?title $=$ Weblog\&oldid $=100025>$, besucht am 31.03.07.

2 netbib Weblog: <http://log.netbib.de>, besucht am 31.03.07.

3 buecherei-Wiki, Filmrecherche: <http://wiki.netbib.de/coma/ Katalog2.0>, besucht am 31.03.07.

4 buecherei-Wiki, MozillaFirefoxSearchPlugins: <http:// buecherei.netbib.de/coma/MozillaFirefoxSearchPlugins $>$, besucht am 31.03.07.

5 Heller, Lambert: Thesenpapier zur Fortbildungsveranstaltung „Social Software - Perspektiven für Wissenschaft und Bibliothek": <http://docs.google.com/View?docid=a748gvz5cx 17dgp8mv>; gekürzter Link: <http://snipurl.com/1ebtr>, besucht am 31.03.07. 
das Weblog dazu genutzt wird, erst einmal für sich - und die Mitleserlnnen - Fakten zusammenzutragen, in den Kommentaren werden Anregungen gesammelt und irgendwann wird dann eventuell auch ein Artikel geschrieben. (Auch wenn ich persönlich zumeist wenig Lust verspüre, einen „seriösen“ Aufsatz für eine Fachzeitschrift zu verfassen.)

Durch Einträge in Weblogs ergeben sich sehr schnell Kontakte mit engagierten KollegInnen. Der Informationsaustausch erfolgt oft über die Kommentarfunktion, und nebenbei ist das Schreiben eines Weblogs eine sehr gute Möglichkeit, eine eigene annotierte Bookmarkliste zu erstellen, die gleichzeitig dazu beiträgt, andere KollegInnen zu informieren.

Inzwischen gibt es natürlich auch noch andere Dienste, die Ähnliches sehr viel effizienter leisten, aber (noch) meist nur von ziemlich fortgeschrittenen Bibliothekarlnnen genutzt werden. Dazu gehören unter anderem die „social bookmarking“-Dienste. Der älteste Vertreter dieser Art ist sicher del.icio.us, weitere sind Connotea, CiteULike und bibsonomy.

\section{Was macht netbib aus?}

\section{Die Autorlnnen}

Erstaunt haben andere netbib-AutorInnen und auch ich zur Kenntnis nehmen müssen, dass es selbst langjährigen LeserInnen des Weblogs nicht klar ist, dass netbib von zur Zeit 15 AutorInnen ${ }^{6}$ gefüllt wird, die, je nach Lust und Laune, mehr oder weniger veröffentlichen.

Nachdem ich zunächst alleine geschrieben hatte, suchte ich im November 2001 über die Liste INETBIB eine „Urlaubsvertretung“. Es meldeten sich Klaus Graf, Jürgen Plieninger und Christian Spließ, mit leichten Bedenken, ob sie mit dem „System Weblog“ zurecht kommen würden. Aber da das so genannte „Posten“ eines Eintrages genauso einfach wie das Verfassen einer Mail über einen Webmail-Account ist, bedurfte es nur einer sehr kurzen Einweisung und die Kollegen legten los.

Die anderen MitschreiberInnen stießen nach und nach dazu. Meist begann es damit, dass sie uns als regelmäßige Leserlnnen auf interessante Neuigkeiten aufmerksam machten. Kam das dann mehrfach vor, bat ich sie darum, doch selbst ins Weblog zu schreiben, was aber zunächst meist mit den Worten „ich weiß ja gar nicht, wie das funktioniert" abgelehnt wurde.

Ein separates Weblog zum Üben nahm die Hemmschwelle, und meist wurde nach 2-3 Testeinträgen ins echte netbib Weblog gepostet. Natürlich gab es auch einige, die diese „Einarbeitung“ nicht benötigten.

Die Überraschung über das unglaublich einfache Veröffentlichen mit dem Werkzeug Weblog stellen wir auch immer wieder auf den hbz-Workshops und anderen Veranstaltungen zum Thema fest.

\section{Die netbib-Wikis}

Anfang 2004 wurde auf netbib das erste bibliothekarische Wiki installiert, das netbib-Wiki ${ }^{7}$. Es sollte zunächst dazu dienen, Erfahrungen mit diesem neuen Werkzeug zu machen. Schnell nutzten es KollegInnen für ihre speziellen Linksammlungen oder auch als Ablageplatz für noch im Entstehen begriffene Texte. Das Werkzeug wurde für gut befunden.

Als die Kollegen Antonius Gusik und Jochen Dudeck (beide aus öffentlichen Bibliotheken) dann bei ihren zuständigen Institutionen um Einrichtung eines eigenen Wikis baten, stießen sie auf Widerstände, die viele KollegInnen auch heute noch zu hören bekommen: „Was ist denn das?", „Das brauchen wir nicht", „Was ist mit der Sicherheit?", „Da kann jeder einfach so reinschreiben?!“. Welche Probleme sich auftun, wenn engagierte KollegInnen auf der Suche nach einem Platz für ihre Dateien sind, um sie der Bibliotheksöffentlichkeit zu präsentieren, beschreibt auch Katrin Sauermann in ihrer Diplomarbeit: „Eine Anfrage beim FachstellenServer ergab, dass,Forum ÖB_multikulturell' einen Fachbereich für Multikulturelle Bibliotheksarbeit auf dem Server erhalten könnte, was jedoch die Bildung einer Facharbeitsgruppe voraussetzt"8.

So wurde netbib unkomplizierte Heimat für das buechereiWiki, welches inzwischen von vielen KollegInnen intensiv genutzt wird. Zu den beliebtesten Seiten zählt die Seite OEB Multikulturell aktuel/9 ${ }^{9}$ die Arbeitshilfen für die Multikulturelle Bibliotheksarbeit bietet. Auch die Seite Filmrecherche ${ }^{10}$ von Peter Delin (ZLB Berlin) mit umfangreichen Informationen zur weltweiten Recherche nach Filmen ist gut besucht und vielfach verlinkt.

Netbib arbeitet mit CoMa-Wiki (nach dem Entwickler Costal Martignier), dessen Entwicklung inzwischen von Costal eingestellt wurde, unter anderem aus Frust über die kaum noch beherrschbare Spam-Schwemme im Netz. Die heute bevorzugte Software MediaWiki ${ }^{11}$ war damals noch zu komplex in der Installation.

Aber vielleicht hätte sich aus heutiger Sicht auch für einige Themen eher ein eigenes Weblog als ein Wiki angeboten.

\section{Der netbib-Podcast}

Seit September 2006 erscheint wöchentlich der netbibPodcast. In dieser Audiodatei werden von Christian Spließ die interessantesten und wichtigsten Einträge des Weblogs noch einmal kurz zusammengefasst und meist auch kommentiert und angereichert. Der Podcast ist natürlich als RSS-Feed abonnierbar, aber wir haben auch zu jedem Podcast-Eintrag einen kleinen Player mit eingebaut,

6 netbib AutorInnen: <http://log.netbib.de/cosmos/>, besucht am 31.03.07.

7 netbib-Wiki: <http://wiki.netbib.de/coma/StartSeite>, besucht am 31.03.07.

8 Sauermann, Katrin: Impulse aus dem Ausland für die multikulturelle Bibliotheksarbeit in der BRD - Darstellung und Erprobung ausgewählter Konzepte, Studiengang Bibliotheks- und Medienmanagement der Fachhochschule Stuttgart - Hochschule der Medien 2005, S. 101 <http://digbib. iuk.hdm-stuttgart.de/epub/volltexte/2005/465/>, besucht am 31.03.07.

9 buecherei-Wiki, OeBmultikulturellAktuell: <http:// buecherei.netbib.de/coma/OeBmultikulturellAktuell>, besucht am 31.03.07.

10 buecherei-Wiki, Filmrecherche: <http://buecherei.netbib.de/ coma/Filmrecherche>, besucht am 31.03.07.

11 MediaWiki, Startseite: <http://www.mediawiki.org/wiki/ MediaWiki>, besucht am 31.03.07. 


\section{netbib weblog}

Weblog mit (haupts?chlich) bibliothekarischen Links

$$
\text { :Startseite }
$$

Abb.1: netbib2001

\section{Freitag, August 3}

Auch in Gomaringen wird schon auf EDV umgestellt

Vor einem Monat hat die umstellung der Bibliothek auf EOV begonnen. Bis die Leser davon etwas haben, wird allerdings noch ein Jahr vergehen. Reutlinger General-Anzeiger

| link | 0 comments

45-Labels

F?r Freunde alter Singles: Abbildungen von Schallplattenetiketten: NoRelevance - Really cool visual junk.

| link | 0 comments

\section{Donnerstag, August 2}

\section{CDRS}

In dem September-Heft vom PC-Magazin ist unter "A.ktuelle Nachrichten" ein kurzer Text zu dem CDRS-Projekt der Library of Congress/OCLC abgedruckt. Sehr sch?n finde ich die Aussage: "Das Projekt soll Bibliothekaren auch in der vom Internet dominierten Zeit eine Daseinsberechtigung geben"
BIBLIOTHEK/AUSKUNFT

TUB | OPAC

KVK

DVB

Toolbox

ZDB

inetbib

links 4help

offizz

$\therefore$ BIBLIOTHEK WEBLOGS

librarian.net

library_geek

library stuff

library planet

rogue librarian

newpages

newbreedlibrarian

abada abada

VAS \& News Desk

weitere logs... mit dem man sich den Inhalt direkt auf der netbib-Seite anhören kann.

Gedacht sind Podcasts aber dafür, um auf mobile Geräte automatisch heruntergeladen und dann, z. B. auf dem Weg zur Arbeit, angehört zu werden. Wer jetzt „wieder so'n neumodischer Kram" denkt, sollte beim nächsten Mal auf der Straße einfach mal genauer auf den omnipräsenten Knopf im Ohr achten. Wenn man weiß, dass fast alle öffentlichen Rundfunkanstalten Podcasts und sogar Video-Podcasts als normalen Service im Angebot haben, wundert man sich natürlich nicht weiter, dass auch deutsche Bibliotheken dieses Medium testen. Die Universitätsbibliothek der Technischen Universität Hamburg Harburg bietet seit letztem Jahr einen Podcast an, und auch die UB der Universität Dortmund plant in Kürze den „Regelbetrieb“. Jüngster Vertreter dieser Riege ist die Zentralbibliothek der Medizin ${ }^{12}$ der Universitäts- und Landesbibliothek Münster.

\section{Der netbib-Newsletter}

Eigentlich ein ziemlicher Anachronismus, aber offensichtlich für über 600 KollegInnen unverzichtbar: Der netbib-Newsletter. Seit März 2002 sind 210 Ausgaben erschienen. Im Newsletter werden einmal wöchentlich die Einträge aus netbib - nach Kategorien geordnet - zusammengefasst und per E-Mail versandt. Ursprünglich war dies nur ein Test mit einem Newsletter-Skript, der lange Zeit mit ca. 30-40 AbonnentInnen vor sich hin lief.
Als der Newsletter aber von einigen KollegInnen relativ euphorisch in der INETBIB-Liste besprochen wurde, stieg die Zahl der AbonnentInnen rasant. Seit ca. drei Jahren sind es über 600. Alle Versuche, den Newsletter einzustellen, scheiterten an den Einsprüchen der AbonnentInnen. Ich hatte auf die Redundanz und auf die alternativen Möglichkeiten des Lesens hingewiesen (Original, per RSS, nur bestimmte Kategorien per RSS, die Möglichkeit, einmal täglich die Einträge per Feedblitz ${ }^{13}$, ein Dienst, der Blogeinträge per E-Mail verschickt, zu beziehen...), aber es scheint eine lieb gewordene Gewohnheit zu sein, am Montag morgen die Einträge der letzten Woche schön nach Kategorien geordnet zu lesen. Und so wird dieser Service erst einmal weitergeführt.

\section{Das medinfo-Weblog}

Das medinfo-Weblog von Dr. Oliver Obst ist ein Teil von netbib, der aber von Oliver Obst völlig autonom betrieben wird. Entstanden ist das medinfo-Weblog aus einem

12 Zentralbibliothek Medizin der ULB Münster, Aktuelle Neuigkeiten: <http://cgi.uni-muenster.de/exec/ZBMed/news. php?newsid=366>; gekürzter Link: <http://snipurl.com/ 1ebtw>, besucht am 31.03.07.

13 netbib Weblog als E-Mail abonnieren: <http://www.feedblitz. com/f/?Sub=91835>, besucht am 31.03.07. 
kleinem Workshop, den ich 2003 auf einer AGMB-Tagung in Münster abgehalten hatte.

Offensichtlich war das Ergebnis so überzeugend, dass der geschätzte Kollege gleich auf einem freien Hoster (twoday.net) einen ersten Versuch startete, dabei aber relativ schnell an Grenzen stieß. Da der netbib-Server damals noch nicht sonderlich belastet war, wurde dort eine Subdomain medinfo.netbib.de eingerichtet, und dann begann auch schon die Erfolgsgeschichte von medinfo. Diese ist aber ein besonderes Thema, über das Oliver Obst in einem eigenen Artikel in diesem Heft berichtet.

\section{Technik Der Start mit Blogger}

Weblogs und ich trafen Ende 2000 aufeinander. Nachdem ich erst mal begriffen hatte, dass Weblogs nichts mit den so genannten Server-Logs (die die Aktivitäten auf einem Webserver mitschreiben) zu tun haben, richtete ich mir bei Blogger einen Account ein. Sehr praktisch fand ich damals schon die Möglichkeit, Blogger nur als Eingabeschnittstelle zu nutzen und die Dateien auf dem eigenen Server zu hosten.

Der größte Nachteil aus bibliothekarischer Sicht war natürlich das Fehlen von Erschließungsmöglichkeiten. So gibt es bei Blogger z. B. erst seit kurzem die Möglichkeit, Tags zu verwenden. Damals war noch keinerlei Kategorisierung der Einträge möglich.

\section{Der Umstieg auf b2}

Nachdem immer leistungsfähigere Skripte für Weblogs auftauchten, entschied ich mich für den Umstieg auf b2. Zwar konnten die alten Kommentare nicht übernommen werden, da diese auf einem Fremdhoster lagen, aber die Übernahme der Datensätze klappte einwandfrei.

b2, das von Michel Valdrighi programmiert wurde, bot folgende Vorteile:

- Eine Suchfunktion, die diesen Namen verdiente

- einfachere Administration

- integrierter XML/RSS-Feed

- komfortablere Oberfläche für das Einbringen der Beiträge

- Kategorien waren nun möglich.

\section{Und schließlich Wordpress}

Michel Valdrighi verlor das Interesse an b2, und so begann unter anderem Matt Mullenweg damit, Wordpress als Weiterentwicklung von b2 ins Leben zu rufen. netbib folgte dieser Entwicklungslinie im März 2004. Die Unterstützung bei der Umstellung auf das neue OpenSource-Produkt durch Matt Mullenweg, den Chefentwickler der (inzwischen) weltweit erfolgreichsten WeblogSoftware, war erstklassig. Da es unbedingt erforderlich war, die alten Einträge mitzunehmen, fragte ich bei Matt an, ob er nicht den Umstieg durchführen könnte. Auf meine Anfrage „...l would like to ask if its possible that you (or a member of the development team) can check my working b2 install and convert it to wordpress..." kam sofort die Antwort "I'd be happy to help you out. How should I access the database and files? --Matt". Kurz nach Zusendung der erforderlichen Daten war die
Umstellung durch Matt erfolgt. Die weiteren Updates auf neue Versionen wurden dann von mir durchgeführt.

\section{Wenn der Server hinter der Idee zurückbleibt}

Im Laufe der Jahre erhielt das netbib immer mehr Zuspruch: Leider nicht nur von vielen interessierten Bibliothekaren und Bibliothekarinnen, sondern auch durch automatisierte Kommentar- und Trackbackspammer. Auf dem Server des Anbieters, auf dem 50 Präsenzen liefen, kam es mitunter soweit, dass netbib $90 \%$ der Ressourcen verschlang. Unser Hoster sah sich dadurch gezwungen, einen Wechsel auf einen teureren Tarif vorzuschlagen. Ein entsprechend ausgelegter Server hätte 50 bis 60 Euro pro Monat gekostet. Die Rettung kam aus Dortmund. Das DV-Team der Universitätsbibliothek bot einen ausrangierten Rechner als Ersatz an, der auch ein gutes Jahr lang lief und dann, Anfang 2007, gegen einen noch schnelleren Server ausgetauscht wurde.

An dieser Stelle noch einmal herzlichen Dank für den fantastischen Support!

\section{Häufige Fragen zu netbib}

Nicht vorenthalten möchte ich Ihnen an dieser Stelle die häufig gestellten und ebenso häufig beantworteten Fragen zum netbib weblog:

\section{Was sind eigentlich Weblogs?}

Ein Weblog ist eine Webseite,

- die chronologisch sortiert ist (aktuellster Eintrag oben)

- meist täglich aktualisiert wird

- eine hohe Linkdichte besitzt

- in Kategorien eingeteilt ist (manchmal)

- eine Archiv- und Suchfunktion hat

- oft eine Kommentarfunktion hat

- einen RSS-Feed anbietet.

Wann schreibt Ihr das eigentlich alles, müsst Ihr nicht arbeiten?

Das ist die häufigste Frage, die man als Weblogschreiberln gestellt bekommt. Viele MitautorInnen (ich eingeschlossen) gehen so vor:

- Andere Weblogs werden - immer auf der Suche nach neuen Informationen - meist außerhalb der Arbeitszeit gelesen.

- Das Lesen wird durch lokal installierte Feedreader vereinfacht. Diese bieten mehr Optionen als z. B. der Webdienst Bloglines und vereinfachen die Weiterverarbeitung von Einträgen aus anderen Weblogs.

- Findet man interessante Links, z. B. durch einen Blick in das Netzwerk von KollegInnen bei del.icio.us, wird der Eintrag per Mausklick übernommen und gegebenenfalls später genauer begutachtet.

- Weblog-Einträge werden oft als Entwurf gespeichert und erst später terminiert veröffentlicht. (Dies kann bei KollegInnen vor Ort schon mal Verwunderung auslösen). Auch das Speichern als Entwurf ist durch zwei Mausklicks erledigt, bei Wordpress erledigt das ein Bookmarklet.

- Weitere Bookmarklets (z. B. für del.icio.us, Connotea, LibraryThing usw.) erleichtern die Verarbeitung und Aufbereitung von zu besprechenden Internetadressen. 


\section{NETBIB WEBLOG}

RSS \$Einträge @Kommentare

Der kurioese Bibliotheksbote worinnen zu finden sind allerley newe Zeitungen

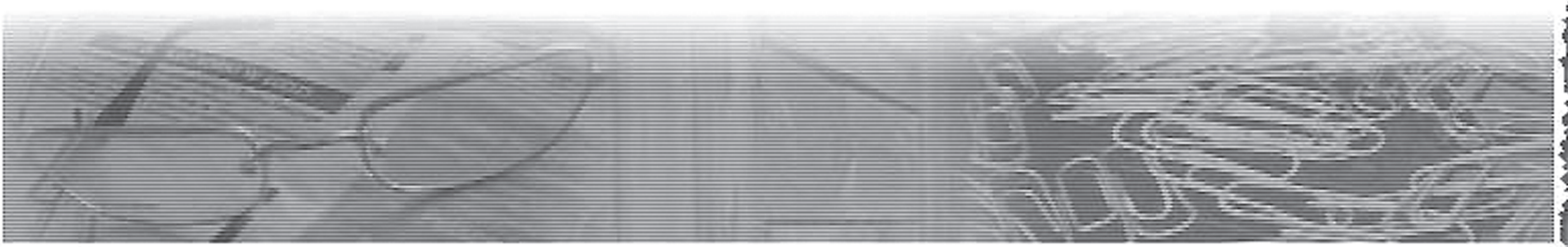

„10 Gründe, warum Du eine öffentliche Bibliothek brauchst ... Jan 29 th, 2007 by FH Edit |

\section{Search}

selbst, wenn Du nie einen Fuss hineingesetzt hast", nennt Margaret ]akubcin von der Jackson County Library.

Hier eine freie Kurzübersetzung des englischen Textes.

1. Bibliotheken sind gut für die Wirtschaft.

Für jeden Dollar, der in die Bibliothek investiert wird, verdienen Unternehmen vor ort 4 Dollar.

2. Bibliotheken sind ein Grundpfeiler der Demokratie.

3. Bibliotheken helfen Kindern bei der Entwicklung der Lesefähigkeit.

4. Öffentliche Bibliotheken fördern Schulen und Studenten.

5 . Bibliotheken sind Vorreiter bei der Informationstechnologie und bieten allen Zugang zum Internet.

6. Bibliotheken beherbergen, schützen und verbreiten Medien, die ein tieferes Verständnis der Welt ermöglichen.

7. Bibliotheken sind ein Schnäppchen. Pro Einwohner kosten sie weniger als zwei gebundene Bücher, einige Monate eines Zeitschriftenabonnements oder

Abb. 2: netbib2007

- Aber natürlich wird auch viel direkt gebloggt. Das ist z. B. bei Neuigkeiten aus der (online-) Tagespresse mit Hilfe des Bookmarklets extrem einfach.

Wie und wann soll ich das denn alles lesen? Ich muss tagsüber arbeiten!

Ganz einfach: natürlich mit einem RSS-Reader, also einem Programm, dass man entweder auf dem eigenen Rechner oder auch als kostenlose „Webapplikation“ nutzen kann. Feeds liest man dann, wenn ein paar Minuten Zeit vorhanden sind. Man hat die aktuellsten Neuigkeiten gebündelt auf einen Blick zur Verfügung. Es ähnelt also einem Blick in die Tageszeitung. Eine ganz praktische Erleichterung ist das Lesen des Planet Biblioblogs ${ }^{14}$, eine von Lambert Heller zusammengestellte Sammlung ${ }^{15}$, die alle deutschsprachigen Weblogs mit dem Fokus auf Bibliothek abdeckt. Bei Aufruf erhält man sofort die letzten Neuigkeiten, sortiert nach Uhrzeit und Datum.

\section{Warum schreibt Ihr das Weblog?}

Für mich war es anfangs ein nützliches Werkzeug für annotierte Bookmarks, inzwischen ist es ein unverzichtbares Kommunikationsmittel geworden. Wichtiger noch: es macht Spaß!

\section{Wer kontrolliert die Einträge?}

Die Einträge werden nicht redaktionell bearbeitet. Jede/r Autor/in entscheidet selbst, was und wie gepostet wird.
Dadurch, dass alle SchreiberInnen alle Einträge editieren können, können z. B. auch Schreibfehler in Einträgen der Mitautorlnnen verbessert werden (was natürlich nur selten vorkommt).

\section{Woher bekommt Ihr die Neuigkeiten?}

Primär durch Auswertung anderer Weblogs, Sichten der bibliothekarischen Fachliteratur und durch verschiedene Alertdienste, die aber jede/r Autor/in für sich selbst bezieht. Meist werden Suchergebnisse per RSS (z. B. von Google News oder auch Technoratiabfragen mit dem Suchwort „bibliothekar" oder Ähnlichem) oder auch per Mail (z. B. Google Alerts ${ }^{16}$ ) bezogen und ,weiterverarbeitet“. Vollständigkeit ist nicht angestrebt.

14 Heller, Lambert: Plante Biblioblogs, deutschsprachige Biblioblogs: <http://www.mysyndicaat.com/myfeed/blog/default/ Lambert_Biblioblogs>; gekürzter Link: <http://snipurl.com/ 1ebuc>, besucht am 31.03.07.

15 LIS Wiki, deutschsprachige bibliothekarische Weblogs: <http://liswiki.org/wiki/Weblogs___Non-English\#German_ .28Deutschsprachig.29>; gekürzter Link: <http://snipurl. com/1ebu0>, besucht am 31.03.07.

${ }^{16}$ Google Alerts: <http://www.google.de/alerts?hl=de>, besucht am 31.03.07. 


\section{Wie viele Leserlnnen hat netbib?}

Die Frage ist - wie üblich wenn es um die Messung von Nutzerfrequenzen geht - nicht ganz einfach zu beantworten.

Nach Auskunft der Kollegen vom DV-Team der UB Dortmund verursacht netbib (inkl. Medinfo-Weblog) pro Tag 1,2 GB ausgehenden Traffic.

Wenn man den verschiedenen Zählern trauen darf, dann hat netbib zwischen 700 bis 1000 LeserInnen pro Tag. Von denen wird aber ungefähr die Hälfte über Suchmaschinen auf netbib geleitet. Google kommt einmal am Tag vorbei und holt sich die Inhalte von beiden Weblogs ab. Dadurch sind die neuesten Einträge auch täglich bei Google indiziert.

Über RSS informieren sich über 1100 LeserInnen über die Neuigkeiten auf netbib. Weitere 600 haben den netbib-Newsletter abonniert.

\section{Wo wird netbib gehostet?}

Die beiden Weblogs netbib und das medinfo-Weblog werden seit Mitte 2006 auf einem Rechner der Universitätsbibliothek Dortmund gehostet. Alle anderen Angebote wie die Wikis liegen bei einem kommerziellen Host auf.

\section{Was verursacht netbib für Kosten?}

Die UB Dortmund beherbergt uns (medinfo-Weblog und netbib Weblog) kostenlos. Für die restlichen netbib-Dienste (wikis ...) fallen die Kosten für den Host an. Hier würde man mit einem Account für etwa 5 Euro/Monat auskommen (wenn ich nicht inzwischen aus verschiedenen Gründen einen teureren Account gebucht hätte).

\section{Die Nebeneffekte}

Was hat sich durch das Schreiben und Lesen von netbib und anderen bibliothekarischen Weblogs verändert?

\section{Praxistaugliche neue Dienste}

Durch das Schreiben des Weblogs und das Nutzen der Erfahrungen der - anfangs meist amerikanischen - KollegInnen haben wir in der TUB HH (das ist die Universitätsbibliothek der TU Hamburg-Harburg) diverse Möglichkeiten neuer Dienstleistungen kennen gelernt, ausprobiert und eingeführt.

Informationen aus Artikeln der deutschen Fachliteratur dagegen zeichnen sich meist dadurch aus, dass mit wohl gesetzten Worten von grundsätzlich erfolgreichen Projekten aus der Bibliotheksszene berichtet wird. Kritische Artikel zu Projekten finden sich eher spärlich. Was fehlt, sind Berichte von Problemen bei oder nach der Einführung von Dienstleistungen oder Webangeboten. Bei den Weblogs der amerikanischen KollegInnen fiel es mir sofort auf: Sie waren sehr schreibfreudig und sparten auch nicht mit Kritik an Webauftritten und Katalogsystemen, und auch die Geschäftspolitik mancher Firmen von Bibliothekssoftware wurde sehr kritisch beleuchtet.

Natürlich bleibt es nicht bei der Kritik, sondern es werden auch sehr hilfreiche Tipps, Workarounds und andere Hilfestellungen in den Weblogs gegeben.

\section{Open Source Software funktioniert}

Wie schon beschrieben, habe ich sehr gute Erfahrungen mit Open Source Programmen und -Programmieren ma- chen können. An der TUB HH setzen wir nach Möglichkeit Open Source Programme für unsere Dienste ein. Angefangen beim Content Management System AWF ${ }^{17}$ über die Chatauskunft mit RAKIM ${ }^{18}$ (RAKIM ist ein ChatSystem, das von Rob Casson an der University of Miami entwickelt wurde) und das Ticketsystem osTicket ${ }^{19}$ (das inzwischen unter dem Namen eTicket ${ }^{20}$ weiterentwickelt wird), das wir für die Beantwortung von Kundenanfragen seit über zwei Jahren im Einsatz haben, und das auch von dem Dienst „Fragen Sie Hamburger Bibliotheken“21 genutzt wird, bis zu unserem Wiki im Intranet haben wir sehr gute Erfahrungen mit dem „Support“ bei Problemen gemacht. Sowohl Rob Casson (RAKIM), Michael Mayer (AWF) als auch die Entwickler von osTicket halfen mit Rat und Tipps weiter. Außerdem haben eigentlich alle Open Source Projekte Foren, in denen um Rat gefragt werden kann. Das funktioniert oft deutlich effizienter als der Support von kommerziellen Anbietern.

Da heute eigentlich alle Open Source Projekte und Projektentwickler auch Weblogs führen, ist es oft so, dass durch die automatischen Vernetzungsfunktionen wie Trackbacks oder auch nur Verlinkung, Entwickler auf die im Weblog beschriebenen eigenen Probleme mit der Software aufmerksam werden und reagieren.

\section{Freiwillige Projekte sind oft erfolgreich}

Wir informieren in netbib über diverse bibliothekarische Projekte; man bekommt dadurch einen Überblick, was gerade läuft, was erfolgreich und was eher nicht so gut klappt.

Beispiele für teilweise sehr erfolgreiche „bibliothekarische“ Projekte von Nichtbibliothekarlnnen gibt es einige, z. B. das von Tim Spalding entwickelte LibraryThing. LibraryThing hat inzwischen weltweit sehr viele Nutzerlnnen, darunter auch diverse Bibliotheken. Dieser „Katalog“ funktioniert nämlich so, wie die normalen NutzerInnen es z. B. von Google und Amazon gewohnt sind. Einfache Bedienung, keine komplizierten Suchmasken, Bewertungssystem, Recommenderfunktionen, Abbildungen der Buchumschläge und vieles mehr.

Natürlich liegt auch den meisten (geförderten) Projekten eine sinnvolle Intention zugrunde, aber viel zu oft wird nach dem ewig gleichen Muster vorgegangen, meist wird versucht, das Rad neu zu erfinden. Mir kommt es dabei so vor, dass auf das durch neue Webtechnologien dramatisch veränderte Nutzerverhalten in den letzten Jahren relativ wenig Rücksicht genommen wird und auch der Blick über den Tellerrand zu häufig fehlt.

Das liegt unter anderem natürlich daran, dass anscheinend viele KollegInnen in einer Art Elfenbeinturm sitzen, nur die

17 Open Source Content Management System AWF: <http:// www.awf-cms.org/>, besucht am 31.03.07.

18 RAKIM: A Knowledge Instant Messenger: <http://rakim. sourceforge.net/>, besucht am 31.03.07.

19 Open Source Support Ticket System osTicket: <http://www. osticket.com/>, besucht am 31.03.07.

20 eTicket is a PHP-based electronic (open source) support ticket system based on osTicket: <http://eticket.sourceforge. net/>, besucht am 31.03.07.

21 „Fragen Sie Hamburger Bibliotheken“: <http://www.tub. tu-harburg.de/mailinfo/virtuelle_auskunft.php?cat=9>, besucht am 31.03.07. 
Fachliteratur rezipieren und sich dadurch erfolgreich vom aktuellen Stand der Technik im Netz ausschließen.

So habe ich herzhaft geschmunzelt, als ich im Projektbericht zur Einführung einer „Virtuelle(n) Auskunft im SWB“22 gelesen habe „Hierbei konnte festgestellt werden, dass die grundsätzlichen Funktionen einer über Webanwendungen organisierten eMail Anfrage- und Weiterleitung inklusive Knowledge Base seit Jahren in Open Source Projekten (sog. bugtracker, issue tracker) entwickelt werden $^{\text {"23 }}$ (Hervorhebung von mir).

Bei der Einführung der „Hamburger Auskunft“, die glücklicherweise etwas unter Zeitdruck stand, haben wir uns die normalen Projektschritte (,Kick Off Meeting“, Sichtung, Pflichtenheft, Umfragen, Evaluation, Ergebnis) gespart und einfach auf ein bewährtes System gesetzt. Bis jetzt gab es noch keine Probleme, die nicht durch das Know How unseres Kollegen Oliver Marahrens gelöst werden konnten.

Auch die Chat-Software RAKIM wird, mit der vom Kollegen Heiko Weier zur Verfügung gestellten deutschen Sprachdatei, von den meisten deutschen Bibliotheken ${ }^{24}$, die eine solche Auskunft anbieten, erfolgreich eingesetzt.

Auf den netbib-Wikis laufen einige kleine Projekte von KollegInnen, und durch die über netbib entstandenen Kontakte findet eine recht erfolgreiche unbürokratische Zusammenarbeit mit anderen KollegInnen aus dem Inund Ausland statt.

Nach meinen Erfahrungen mit Projekten wird meist eben nicht danach geschaut, ob es Open Source Anwendungen gibt, sondern es wird versucht, kommerzielle Software so hinzubiegen, dass es passt. Unter anderem dadurch ist meiner Meinung nach z. B. das Projekt „Forum Benutzung", dem eine wirklich sehr gute Idee zu Grunde liegt - „Unter diesem Titel arbeitet die Dienstleistungskommission (des DBV) an einer Online-Datenbank, die beispielhafte Dienstleistungen öffentlicher und wissenschaftlicher Bibliotheken in Deutschland beinhalten soll sowie Literaturquellen sichtet und verzeichnet" - nicht so erfolgreich geworden, wie es wünschenswert gewesen wäre.

Es gibt für alle denkbaren Bereiche auch Open Source Software, und dieses manchmal noch vorherrschende Denken, „Ich bezahle lieber, dann habe ich jemanden der verantwortlich ist", ist extrem kurzsichtig, wie diverse Beispiele beweisen. Auch kommerzielle Firmen gehen in Konkurs, und will man - banales Beispiel aus der Praxis - zusätzlich einen RSS-Feed von seinem Content Management System generieren lassen, kostet das bei einem normalen Anbieter natürlich extra.

\section{Fazit}

Zum Thema „Fachblogs" gibt es inzwischen natürlich auch wissenschaftliche Untersuchungen. Bibliothekarische Fachblogs hat die Kollegin Heidi Stieger in ihrer Diplom-

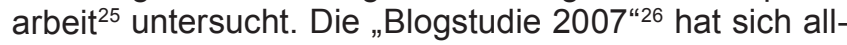
gemeiner mit dem Thema befasst und konstatiert: „Fachblogs von Experten und Blogs von Medien/Journalisten sind wichtige Impulsgeber für neue Ideen, Themen und Meinungen“27, während „Blognutzer (...) mehrheitlich investigative Multiplikatoren (sind) - Konsumenten, die mehr wissen wollen, Informationen aktiv weitergeben und gut vernetzt sind" 28

Wie man hört und auch selber erfährt, leiden viele KollegInnen an der Informationsüberflutung. Manche sind so überfordert, dass sie es aufgegeben oder nie versucht haben, die wichtigste deutsche Mailingliste zum Thema „Internet und Bibliotheken“, INETBIB ${ }^{29}$, zu lesen. Natürlich kommt immer wieder mal etwas, was einen nicht interessiert und vielleicht auch einiges, was einen ratlos zurücklässt über diese Liste, aber schließlich verstehen sich ja BibliothekarInnen auch als InformationsspezialistInnen?!

Da sollte man es doch eigentlich erwarten können, dass diese über "skills" und auch Werkzeuge verfügen, mit Informationen, woher auch immer, umzugehen und diese zu filtern und einzusortieren.

Durch das Schreiben und auch das Lesen im netbib Weblog habe ich sehr viele neue Impulse und Ideen für die praktische Arbeit in der Bibliothek bekommen (vielen Dank an die anderen netbib AutorInnen!!), den Einsatz von neuen Techniken und Werkzeugen gelernt, und es ist zu vielen wertvollen Kontakten - auch mit Nichtbibliothekarlnnen - gekommen.

Der große Vorteil von Fachblogs ist für mich die authentische und subjektive Berichterstattung. Durch den Schreibstil lernt man mit der Zeit die AutorInnen kennen und schätzen. Man bekommt auch kontroverse Meinungen zu lesen, was ich sehr hoch bewerte, da eigentlich alle Autorlnnen deutscher bibliothekarischer Weblogs nicht anonym, sondern unter ihrem echten Namen schreiben.

Abschließend meinen Dank an die anderen netbib-AutorInnen, die mit ihren Interessen viele verschiedene Felder der bibliothekarischen Arbeit abdecken, mich und andere an diesem Wissen teilhaben lassen, und an die vielen Leserlnnen, die uns manchmal per Mail und oft in den Kommentaren ihre Meinungen und Erfahrungen mitteilen!

\section{Anschrift des Autors:}

\section{Edlef Stabenau}

Universitätsbibliothek der Technischen

Universität Hamburg-Harburg

D-21071 Hamburg

Tel.: +49 40428782230

Fax: +4940428782248

E-Mail: stabenau@tu-harburg.de

Website des netbib-Projekts: <http://netbib.de/>

22 Vorstudie zum Projekt, Virtuelle Auskunft im SWB' Endbericht: <http://titan.bsz-bw.de/cms/entwickl/virtausk/ VirtuelleAuskunftBerichtVorstudie.pdf/file_view>; gekürzter Link: <http://snipurl.com/1ebu4>, besucht am 31.03.07.

${ }^{23}$ Ebd., S. 15.

24 Stabenau, Edlef: Übersicht über Chatangebote deutscher Bibliotheken: <http://www.bibliothekar.de/content/view/15/26/>, besucht am 31.03.07.

25 Stieger, Heidi: Fachblogs von und für Bibliothekarlnnen Nutzen, Tendenzen. Mit Fokus auf den deutschsprachigen Raum. In: Churer Schriften zur Informationswissenschaft 16 (2007) <http://www.iudchur.net/fileadmin/uploads/pdf/ csi/CSI_16_Stieger.pdf>, besucht am 31.03.07.

${ }^{26}$ Blogstudie 2007, Informationssuche im Internet - Blogs als neues Recherchetool <http://www.blogstudie2007.de/>, besucht am 31.03.07.

27 Blogstudie 2007 (Anm. 24), S. 9.

${ }^{28}$ Ebd., S. 7.

${ }^{29}$ Homepage der Mailingliste Inetbib: <http://www.inetbib.de>, besucht am 31.03.07. 